CORRESPONDENCE.

\title{
ON THE NUMBER OF YEARS IN WHICH PREMIUMS AMOUNT TO TWICE THE TOTAL SUM PAID.
}

To the Editors of the Journal of the Institute of Actuaries.

DeAR SirS, - In furtherance of a plan which had proved effective in securmg proposals for assurance, a New Busmess Superintendent of this City sought to discover a method by which he might readily calculate the number of years in which premiums paid on a life assurance policy would at any given rate of interest amount to twice the total of such premiums. By simple experiment with tables of the values of $s_{n]}$ he ascertained that, if either the number of years or the interest rate per-cent were given, the other could be found by dividing 125 by the given value.

I am not aware that this simple formula, which gives a remarkably close approximation to the true result, has ever been published. I venture, therefore, to bring it under your notice and to furnish the following algebraical rerification 
To prove that if $n=125 / 100 i$, or $100 i=125 / n, s_{n+1}-1=2 n$ approximately.

$$
\begin{aligned}
& s_{n+1}-1=\frac{(1+i)^{n+1}-1}{i}-1 \\
& =\frac{1+(n+1) i+\frac{(n+1) n}{2} \cdot i^{2}+\frac{(n+1) n(n-1)}{23} \cdot i^{3}+\ldots-1}{i}-1 \\
& =(n+1)+\frac{(n+1) n}{2} i+\frac{(n+1) n(n-1)}{2.3} i^{2}+\ldots-1 \\
& =n+\frac{n^{2}+n}{2} \cdot \frac{1 \cdot 25}{n}+\frac{n^{3}-n}{6}\left(\frac{1 \cdot 25}{n}\right)^{2}+\frac{n^{4}-2 n^{3}-n^{2}+2 n}{24}\left(\frac{1 \cdot 25}{n}\right)^{3} \\
& +\frac{n^{5}-5 n^{4}+5 n^{3}+\ldots}{120}\left(\frac{1 \cdot 25}{n}\right)^{4}+\frac{n^{6}-9 n^{5}+25 n^{4} \ldots}{720}\left(\frac{1 \cdot 25}{n}\right)^{5}+\ldots \\
& =n(1+\cdot 625+\cdot 260+\cdot 082+\cdot 020+\cdot 004+\cdot 001+\ldots) \\
& +(\cdot 625+0-\cdot 163-102-\cdot 038-\cdot 009 \ldots) \\
& +\frac{1}{n}(-\cdot 260-\cdot 082+\cdot 102+\cdot 105+\cdot 049 \ldots) \\
& +\ldots \ldots \\
& =n \times 1 \cdot 99 \ldots+\cdot 31 \ldots+\frac{1}{n}(-\cdot 09 \ldots)+\ldots \\
& =2 n \text { (approx.) }
\end{aligned}
$$

Yours faithfully,

459, Collins Street, Melbourne,

P. D. TOUZEL.

I September 1925.

[Nearly the same result may be obtained by taking $s_{n+1}$, i.e., $1+(1+i)+(1+i)^{2}+\ldots+(1+i)^{n}$ as roughly equal--on the analogy of Simpson's formula-to $\frac{1}{6}(n+1)\left\{1+4(1+i)^{\frac{1}{2} n}+(1+i)^{n}\right\}$. We then have, if $s_{n+1}-1=2 n, \quad(1+i)^{n}+4(1+i)^{\frac{1}{2} n}+4=15\{1-2 / 5(n+1)\}$ whence, approximately, $(1+i)^{\frac{1}{n} n}+2=3.873\{1-1 / 5(n+1)\}$;

$$
\frac{1}{2} n \log _{e}(1+i)=\log _{e} 1 \cdot 873+\log _{e}\{1-2 \cdot 07 / 5(\hat{\imath}+1)\}
$$

$=\cdot 6275-2 / 5(n+1) ;$ and $i=\frac{1 \cdot 255}{n}-\frac{4}{5 n(n+1)}$-EDS. J.I.A.] 